\title{
Existence and $\beta$-Ulam-Hyers stability for a class of fractional differential equations with non-instantaneous impulses
}

\section{Xiulan Yu*}

\section{"Correspondence:}

xly3028440@126.com

College of Applied Mathematics,

Shanxi University of Finance and

Economics, Taiyuan, Shanxi 030031,

P.R. China

\begin{abstract}
In this paper, we investigate a new class of fractional differential equations with non-instantaneous impulses. We give a suitable formula of piecewise continuous solutions and present the concept of $\beta$-Ulam-Hyers stability. We present existence and $\beta$-Ulam-Hyers stability results on a compact interval.
\end{abstract}

Keywords: fractional differential equations; non-instantaneous impulses; existence; $\beta$-Ulam-Hyers stability

\section{Introduction}

Impulsive fractional differential equations are used to describe many practical dynamical systems in many evolutionary processes models. There are many recent contributions [14] on fractional differential equations with instantaneous impulses of the form

$$
\left\{\begin{array}{l}
{ }^{\mathrm{c}} D_{0, t}^{\alpha} x(t)=f(t, x(t)), \quad t \in[0, T] \backslash\left\{\tau_{1}, \ldots, \tau_{m}\right\}, \\
\Delta x\left(\tau_{k}\right)=I_{k}\left(x\left(\tau_{k}^{-}\right)\right), \quad k=1,2, \ldots, m
\end{array}\right.
$$

where ${ }^{\mathrm{c}} D_{0, t}^{\alpha}$ is the Caputo fractional derivative of the order $\alpha \in(n-1, n), n \in \mathbb{N}, f: J \times \mathbb{R} \rightarrow$ $\mathbb{R}$ and $I_{k}: \mathbb{R} \rightarrow \mathbb{R}$ and $\tau_{k}$ satisfies $0=\tau_{0}<\tau_{1}<\cdots<\tau_{m}<\tau_{m+1}=T, x\left(\tau_{k}^{+}\right)=\lim _{\epsilon \rightarrow 0^{+}} x\left(\tau_{k}+\epsilon\right)$ and $x\left(\tau_{k}^{-}\right)=\lim _{\epsilon \rightarrow 0^{-}} x\left(\tau_{k}+\epsilon\right)$ represent the right and left limits of $x(t)$ at $t=\tau_{k}$, respectively. Here, $I_{k}$ is a sequence of instantaneous impulse operators and it has been used to describe abrupt changes such as shocks, harvesting, and natural disasters.

In general, the classical instantaneous impulses cannot describe some certain dynamics of evolution processes. For example, when we consider the hemodynamic equilibrium of a person, the introduction of the drugs in the bloodstream and the consequent absorption for the body are gradual and continuous process. In fact, the above situation can be characterized by a new case of impulsive action, which starts at an arbitrary fixed point and stays active on a finite time interval. From the viewpoint of general theories, Hernández and O'Regan [5] initially offered a study of a new class of abstract cases of semilinear impulsive differential modeling with no instantaneous impulses and Pierri et al. [6] continued the work and extended the previous results.

However, we note that the absorption of drugs has a memory effect. In fact, fractional calculus provides a powerful tool for hereditary properties on various materials and mem-

( ) 2015 Yu: licensee Springer. This is an Open Access article distributed under the terms of the Creative Commons Attribution License (http://creativecommons.org/licenses/by/4.0), which permits unrestricted use, distribution, and reproduction in any medium, provided the original work is properly credited. 
ory processes $[7,8]$. Motivated by [5-8], we investigate the following new class of impulsive differential equations:

$$
\left\{\begin{array}{l}
{ }^{\mathrm{c}} D_{s_{i}, t}^{\alpha} x(t)=-\lambda x(t)+f(t, x(t)), \quad t \in\left(s_{i}, t_{i+1}\right], i=0,1,2, \ldots, m, \alpha \in(0,1), \lambda \geq 0, \\
x(t)=q+I_{t_{i}, t}^{\gamma} g_{i}(t, x(t))-I_{0, s_{i}}^{\alpha} f\left(s_{i}, x\left(s_{i}\right)\right), \quad t \in\left(t_{i}, s_{i}\right], i=1,2, \ldots, m, \gamma \in(0,1), \gamma \neq \alpha,
\end{array}\right.
$$

where ${ }^{\mathrm{c}} D_{s_{i}, t}^{\alpha}$ is the Caputo fractional derivative of the order $\alpha \in(0,1)$ with the lower limit $s_{i}, 0=s_{0}<t_{1} \leq s_{1} \leq t_{2}<\cdots<t_{m} \leq s_{m} \leq t_{m+1}=T$ are pre-fixed numbers, $f:[0, T] \times \mathbb{R} \rightarrow \mathbb{R}$ is continuous and $g_{i}:\left[t_{i}, s_{i}\right] \times \mathbb{R} \rightarrow \mathbb{R}$ is continuous for all $i=1,2, \ldots, m$ and $q \in \mathbb{R}$. $I_{t_{i}}^{\gamma}, g_{i}$ and $I_{0, s_{i}}^{\alpha} f$ are given by

$$
\begin{aligned}
& I_{t_{i}, t}^{\gamma} g_{i}(t, x(t))=\frac{1}{\Gamma(\gamma)} \int_{t_{i}}^{t}(t-s)^{\gamma-1} g_{i}(s, x(s)) d s, \\
& I_{0, s_{i}}^{\alpha} f\left(s_{i}, x\left(s_{i}\right)\right)=\frac{1}{\Gamma(\alpha)} \int_{0}^{s_{i}}\left(s_{i}-s\right)^{\alpha-1} f(s, x(s)) d s .
\end{aligned}
$$

The Ulam stability problem [9] has attracted many famous researchers. For more details, the readers can refer to good monographs of Hyers et al. [10], Rassias [11], Jung [12], Cădariu [13] and other recent contributions [14-20] in standard normed spaces and [21, $22]$ in $\beta$-normed spaces. As far as we known, neither the existence of a solution nor the Ulam type stability of (1) in $\beta$-normed spaces has been studied. Here, we shall apply the usual methods of analysis and novel techniques in $\beta$-Banach spaces to deal with our problem.

\section{Preliminaries}

To begin with, we present the concept of $\beta$-Banach space.

Definition 2.1 ([14]) Suppose $E$ is a vector space over $\mathbb{K}$. A function $\|\cdot\|_{\beta}(0<\beta \leq 1)$ : $E \rightarrow[0, \infty)$ is called a $\beta$-norm if and only if it satisfies (i) $\|x\|_{\beta}=0$ if and only if $x=0$; (ii) $\|\lambda x\|_{\beta}=|\lambda|^{\beta}\|x\|_{\beta}$ for all $\lambda \in \mathbb{K}$ and all $x \in E$; (iii) $\|x+y\|_{\beta} \leq\|x\|_{\beta}+\|y\|_{\beta}$. The pair $\left(E,\|\cdot\|_{\beta}\right)$ is called a $\beta$-normed space. A $\beta$-Banach space is a complete $\beta$-normed space.

Let $J=[0, T]$ and $C(J, \mathbb{R})$ be the $\beta$-Banach space of all continuous functions from $J$ into $\mathbb{R}$ with the $\beta$-norm $\|x\|_{\beta}:=\max \left\{|x(t)|^{\beta}: t \in J, 0<\beta<1\right\}$ for $x \in C(J, \mathbb{R})$. We also need the piecewise continuous $\beta$-Banach space $P C(J, \mathbb{R}):=\left\{x: J \rightarrow \mathbb{R}: x \in C\left(\left(t_{k}, t_{k+1}\right], \mathbb{R}\right), k=\right.$ $0,1, \ldots, m$, and there exist $x\left(t_{k}^{-}\right)$and $x\left(t_{k}^{+}\right), k=1, \ldots, m$, with $\left.x\left(t_{k}^{-}\right)=x\left(t_{k}\right)\right\}$ with the $P \beta$-norm $\|x\|_{P \beta}:=\sup \left\{|x(t)|^{\beta}: t \in J, 0<\beta<1\right\}$.

Next, we recall some basic concepts of the fractional integral and derivative, and some results as regards fractional differential equations [23].

Definition 2.2 The fractional integral of order $\gamma$ with the lower limit $a$ for a function $f$ is defined as

$$
I_{a, f}^{\gamma} f(t)=\frac{1}{\Gamma(\gamma)} \int_{a}^{t} \frac{f(s)}{(t-s)^{1-\gamma}} d s, \quad t>a, \gamma>0,
$$

provided the right side is point-wise defined on $[a, \infty)$, where $\Gamma(\cdot)$ is the gamma function. 
Definition 2.3 The Riemann-Liouville derivative of order $\gamma$ with the lower limit $a$ for a function $f:[a, \infty) \rightarrow \mathbb{R}$ can be written as

$$
{ }^{L} D_{a, t}^{\gamma} f(t)=\frac{1}{\Gamma(n-\gamma)} \frac{d^{n}}{d t^{n}} \int_{a}^{t} \frac{f(s)}{(t-s)^{\gamma+1-n}} d s, \quad t>a, n-1<\gamma<n .
$$

Definition 2.4 The Caputo derivative of order $\gamma$ for a function $f:[a, \infty) \rightarrow \mathbb{R}$ can be written as

$$
{ }^{\mathrm{c}} D_{a, t}^{\gamma} f(t)={ }^{L} D_{a, t}^{\gamma}\left[f(t)-\sum_{k=0}^{n-1} \frac{t^{k}}{k !} f^{(k)}(a)\right], \quad t>a, n-1<\gamma<n .
$$

Denote $\mathbb{E}_{\alpha}(z):=\sum_{k=0}^{\infty} \frac{z^{k}}{\Gamma(\alpha k+1)}$ and $\mathbb{E}_{\alpha, \alpha}(z):=\sum_{k=0}^{\infty} \frac{z^{k}}{\Gamma(\alpha k+\alpha)}$. By [24], Lemma 2, for any $\lambda \geq$ 0 and $t \in J, \mathbb{E}_{\alpha}(0)=1, \mathbb{E}_{\alpha}\left(-t^{\alpha} \lambda\right) \leq 1, \mathbb{E}_{\alpha, \alpha}\left(-t^{\alpha} \lambda\right) \leq \frac{1}{\Gamma(\alpha)}$.

Lemma 2.5 Let $h: J \rightarrow \mathbb{R}$ be a continuous function. A function $x \in P C(J, \mathbb{R})$ is a solution of the fractional integral equations

$$
\begin{aligned}
x(0)= & x_{0} ; \\
x(t)= & \mathbb{E}_{\alpha}\left(-t^{\alpha} \lambda\right) x_{0}+\int_{0}^{t}(t-s)^{\alpha-1} \mathbb{E}_{\alpha, \alpha}\left(-(t-s)^{\alpha} \lambda\right) h(s) d s, \quad t \in\left(0, t_{1}\right] ; \\
x(t)= & q+I_{t_{i}, t}^{\gamma} g_{i}(t)-I_{0, s_{i}}^{\alpha} h\left(s_{i}\right), \quad t \in\left(t_{i}, s_{i}\right], i=1,2, \ldots, m ; \\
x(t)= & \mathbb{E}_{\alpha}\left(-\left(t-s_{i}\right)^{\alpha} \lambda\right)\left(q+I_{t_{i}, s_{i}}^{\gamma} g_{i}\left(s_{i}\right)-I_{0, s_{i}}^{\alpha} h\left(s_{i}\right)\right) \\
& +\int_{s_{i}}^{t}(t-s)^{\alpha-1} \mathbb{E}_{\alpha, \alpha}\left(-(t-s)^{\alpha} \lambda\right) h(s) d s, \quad t \in\left(s_{i}, t_{i+1}\right], i=1, \ldots, m,
\end{aligned}
$$

if and only if $x$ is a solution of the equation

$$
\left\{\begin{array}{l}
{ }^{\mathrm{c}} D_{s_{i},}^{\alpha} x(t)=-\lambda x(t)+h(t), \quad t \in\left(s_{i}, t_{i+1}\right], i=0,1,2, \ldots, m, \alpha \in(0,1), \\
x(t)=q+I_{t_{i},}^{\gamma} t_{i}(t)-I_{0, s_{i}}^{\alpha} h\left(s_{i}\right), \quad t \in\left(t_{i}, s_{i}\right], i=1,2, \ldots, m, \gamma \in(0,1), \\
x(0)=x_{0} \in \mathbb{R} .
\end{array}\right.
$$

Proof Suppose that $x$ satisfies (2).

For $t \in\left[0, t_{1}\right]$, we consider

$$
{ }^{\mathrm{c}} D_{0, t}^{\alpha} x(t)=-\lambda x(t)+h(t), \quad \text { with } x(0)=x_{0} .
$$

Integrating from 0 to $t$ by virtue of Definition 2.2, one can obtain

$$
x(t)=\mathbb{E}_{\alpha}\left(-t^{\alpha} \lambda\right) x_{0}+\int_{0}^{t}(t-s)^{\alpha-1} \mathbb{E}_{\alpha, \alpha}\left(-(t-s)^{\alpha} \lambda\right) h(s) d s, \quad t \in\left[0, t_{1}\right] .
$$

For $t \in\left(t_{1}, s_{1}\right], x(t)=q+I_{t_{1}, t}^{\gamma} g_{1}(t)-I_{0, s_{1}}^{\alpha} h\left(s_{1}\right)$.

For $t \in\left(s_{1}, t_{2}\right]$, we consider

$$
{ }^{\mathrm{c}} D_{s_{1}, t}^{\alpha} x(t)=-\lambda x(t)+h(t), \quad \text { with } x\left(s_{1}\right)=q+I_{t_{1}, s_{1}}^{\gamma} g_{1}\left(s_{1}\right)-I_{0, s_{1}}^{\alpha} h\left(s_{1}\right) .
$$


Then

$$
\begin{aligned}
x(t)= & \int_{s_{1}}^{t}(t-s)^{\alpha-1} \mathbb{E}_{\alpha, \alpha}\left(-(t-s)^{\alpha} \lambda\right) h(s) d s \\
& +\mathbb{E}_{\alpha}\left(-\left(t-s_{1}\right)^{\alpha} \lambda\right)\left(q+I_{t_{1}, s_{1}}^{\gamma} g_{1}\left(s_{1}\right)-I_{0, s_{1}}^{\alpha} h\left(s_{1}\right)\right) .
\end{aligned}
$$

For $t \in\left(t_{2}, s_{2}\right], x(t)=q+I_{t_{2}, t}^{\gamma} g_{2}(t)-I_{0, s_{2}}^{\alpha} h\left(s_{2}\right)$.

For $t \in\left(s_{2}, t_{3}\right]$, we consider

$$
{ }^{\mathrm{c}} D_{s_{2}, t}^{\alpha} x(t)=-\lambda x(t)+h(t), \quad \text { with } x\left(s_{2}\right)=q+I_{t_{2}, s_{2}}^{\gamma} g_{2}\left(s_{2}\right)-I_{0, s_{2}}^{\alpha} h\left(s_{2}\right) .
$$

So we get

$$
\begin{aligned}
x(t)= & \int_{s_{2}}^{t}(t-s)^{\alpha-1} \mathbb{E}_{\alpha, \alpha}\left(-(t-s)^{\alpha} \lambda\right) h(s) d s \\
& +\mathbb{E}_{\alpha}\left(-\left(t-s_{2}\right)^{\alpha} \lambda\right)\left(q+I_{t_{2}, s_{2}}^{\gamma} g_{2}\left(s_{2}\right)-I_{0, s_{2}}^{\alpha} h\left(s_{2}\right)\right) .
\end{aligned}
$$

Finally, for any $t \in\left(s_{i}, t_{i+1}\right]$, we consider

$$
{ }^{\mathrm{c}} D_{s_{i}, t}^{\alpha} x(t)=-\lambda x(t)+h(t), \quad \text { with } x\left(s_{i}\right)=q+I_{t_{i}, s_{i}}^{\gamma} g_{i}\left(s_{i}\right)-I_{0, s_{i}}^{\alpha} h\left(s_{i}\right) .
$$

Thus,

$$
\begin{aligned}
x(t)= & \int_{s_{i}}^{t}(t-s)^{\alpha-1} \mathbb{E}_{\alpha, \alpha}\left(-(t-s)^{\alpha} \lambda\right) h(s) d s \\
& +\mathbb{E}_{\alpha}\left(-\left(t-s_{i}\right)^{\alpha} \lambda\right)\left(q+I_{t_{i}, s_{i}}^{\gamma} g_{i}\left(s_{i}\right)-I_{0, s_{i}}^{\alpha} h\left(s_{i}\right)\right) .
\end{aligned}
$$

Conversely, one can verify the fact by proceeding the standard steps to complete the rest of proof.

\section{$3 \boldsymbol{\beta}$-Ulam-Hyers stability concept and auxiliary facts}

Let $0<\beta<1, \epsilon>0$. We consider the following inequality:

$$
\left\{\begin{array}{l}
\left|{ }^{c} D_{s_{i}, t}^{\alpha} y(t)+\lambda y(t)-f(t, y(t))\right| \leq \epsilon, \quad t \in\left(s_{i}, t_{i+1}\right], i=0,1,2, \ldots, m, \\
\left|y(t)-q-I_{t_{i}, t}^{\gamma} g_{i}(t, y(t))+I_{0, s_{i}}^{\alpha} f\left(s_{i}, y\left(s_{i}\right)\right)\right| \leq \epsilon, \quad t \in\left(t_{i}, s_{i}\right], i=1,2, \ldots, m .
\end{array}\right.
$$

Then our goal is to find a solution $y(\cdot)$ close to the measured output $x(\cdot)$ and this closeness is defined in the sense of $\beta$-Ulam's type stability as follows.

Definition 3.1 Equation (1) is $\beta$-Ulam-Hyers stable if there exists a real number $c_{f, \alpha, \gamma, \beta, g_{i}}>$ 0 such that for each $\epsilon>0$ and for each solution $y \in P C^{1}(J, \mathbb{R})$ of the inequality (3) there exists a solution $x \in P C^{1}(J, \mathbb{R})$ of (1) with

$$
|y(t)-x(t)|^{\beta} \leq c_{f, \alpha, \gamma, \beta, g_{i}} \epsilon^{\beta}, \quad t \in J .
$$

Remark 3.2 A function $y \in P C^{1}(J, \mathbb{R})$ is a solution of the inequality (3) if and only if there is a number $G$ such that 
(i) $|G| \leq \epsilon$;

(ii) ${ }^{\mathrm{c}} D_{s_{i}, t}^{\alpha} y(t)=-\lambda y(t)+f(t, y(t))+G, t \in\left(s_{i}, t_{i+1}\right], i=0,1,2, \ldots, m$;

(iii) $y(t)=q+I_{t_{i},}^{\gamma} t_{i}(t, y(t))-I_{0, s_{i}}^{\alpha} f\left(s_{i}, y\left(s_{i}\right)\right)+G, t \in\left(t_{i}, s_{i}\right], i=1,2, \ldots, m$.

Remark 3.3 If $y \in P C^{1}(J, \mathbb{R})$ is a solution of the inequality (3) then $y$ is a solution of the following integral inequality:

$$
\left\{\begin{array}{l}
\left|y(t)-q-I_{t_{i}, t}^{\alpha} g_{i}(t, y(t))-I_{0, s_{i}}^{\alpha} f\left(s_{i}, y\left(s_{i}\right)\right) d s\right| \leq \epsilon, \quad t \in\left(t_{i}, s_{i}\right], i=1,2, \ldots, m ; \\
\left|y(t)-\mathbb{E}_{\alpha}\left(-t^{\alpha} \lambda\right) y(0)-\int_{0}^{t}(t-s)^{\alpha-1} \mathbb{E}_{\alpha, \alpha}\left(-(t-s)^{\alpha} \lambda\right) f(s, y(s)) d s\right| \\
\leq \frac{t_{1}^{\alpha}}{\Gamma(\alpha+1)} \epsilon, \quad t \in\left[0, t_{1}\right] \\
\mid y(t)-\int_{s_{i}}^{t}(t-s)^{\alpha-1} \mathbb{E}_{\alpha, \alpha}\left(-(t-s)^{\alpha} \lambda\right) f(s, y(s)) d s \\
\quad-\mathbb{E}_{\alpha}\left(-\left(t-s_{i}\right)^{\alpha} \lambda\right)\left(q+I_{t_{i}, s_{i}}^{\gamma} g_{i}\left(s_{i}, y\left(s_{i}\right)\right)-I_{0, s_{i}}^{\alpha} f\left(s_{i}, y\left(s_{i}\right)\right)\right) \mid \\
\leq \epsilon+\frac{\left(t_{i+1}-s_{i}\right)}{\Gamma(\alpha+1)} \epsilon, \quad t \in\left(s_{i}, t_{i+1}\right], i=1,2, \ldots, m .
\end{array}\right.
$$

In fact, by Remark 3.2 we get

$$
\left\{\begin{array}{l}
{ }^{\mathrm{c}} D_{s_{i}, t}^{\alpha} y(t)=-\lambda y(t)+f(t, y(t))+G, \quad t \in\left(s_{i}, t_{i+1}\right], i=0,1,2, \ldots, m, \\
y(t)=q+I_{t_{i}, t}^{\gamma} g_{i}(t, y(t))-I_{0, s_{i}}^{\alpha} f\left(s_{i}, y\left(s_{i}\right)\right)+G, \quad t \in\left(t_{i}, s_{i}\right], i=1,2, \ldots, m .
\end{array}\right.
$$

Clearly, the solution of (5) is given by

$$
\begin{aligned}
y(t)= & q+I_{t_{i}, t}^{\gamma} g_{i}(t, y(t))-I_{0, s_{i}}^{\alpha} f\left(s_{i}, y\left(s_{i}\right)\right)+G, \quad t \in\left(t_{i}, s_{i}\right], i=1,2, \ldots, m ; \\
y(t)= & \mathbb{E}_{\alpha}\left(-t^{\alpha} \lambda\right) y(0)+\int_{0}^{t}(t-s)^{\alpha-1} \mathbb{E}_{\alpha, \alpha}\left(-(t-s)^{\alpha} \lambda\right)[f(s, y(s))+G] d s, \quad t \in\left[0, t_{1}\right] ; \\
y(t)= & \int_{s_{i}}^{t}(t-s)^{\alpha-1} \mathbb{E}_{\alpha, \alpha}\left(-(t-s)^{\alpha} \lambda\right)[f(s, y(s))+G] d s \\
& +\mathbb{E}_{\alpha}\left(-\left(t-s_{i}\right)^{\alpha} \lambda\right)\left(q+I_{t_{i}, s_{i}}^{\gamma} g_{i}\left(s_{i}, y\left(s_{i}\right)\right)-I_{0, s_{i}}^{\alpha} f\left(s_{i}, y\left(s_{i}\right)\right)+G\right), \\
& t \in\left(s_{i}, t_{i+1}\right], i=1,2, \ldots, m .
\end{aligned}
$$

For $t \in\left(s_{i}, t_{i+1}\right], i=1,2, \ldots, m$, we get

$$
\begin{aligned}
\mid y(t) & -\int_{s_{i}}^{t}(t-s)^{\alpha-1} \mathbb{E}_{\alpha, \alpha}\left(-(t-s)^{\alpha} \lambda\right) f(s, y(s)) d s \\
& -\mathbb{E}_{\alpha}\left(-\left(t-s_{i}\right)^{\alpha} \lambda\right)\left(q+I_{t_{i}, s_{i}}^{\gamma} g_{i}\left(s_{i}, y\left(s_{i}\right)\right)-I_{0, s_{i}}^{\alpha} f\left(s_{i}, y\left(s_{i}\right)\right)\right) \mid \\
\leq & |G|+\left|\frac{1}{\Gamma(\alpha)} \int_{s_{i}}^{t}(t-s)^{\alpha-1} G d s\right| \\
\leq & \epsilon+\frac{1}{\Gamma(\alpha)} \int_{s_{i}}^{t}(t-s)^{\alpha-1} \epsilon d s \\
\leq \epsilon & +\frac{\left(t_{i+1}-s_{i}\right)^{\alpha}}{\Gamma(\alpha+1)} \epsilon .
\end{aligned}
$$

Proceeding as above, we derive that

$$
\left|y(t)-q-I_{t_{i},}^{\alpha} g_{i}(t, y(t))-I_{0, s_{i}}^{\alpha} f\left(s_{i}, y\left(s_{i}\right)\right)\right| \leq|G| \leq \epsilon, \quad t \in\left(t_{i}, s_{i}\right], i=1,2, \ldots, m
$$


and

$$
\begin{aligned}
& \left|y(t)-\mathbb{E}_{\alpha}\left(-t^{\alpha} \lambda\right) y(0)-\int_{0}^{t}(t-s)^{\alpha-1} \mathbb{E}_{\alpha, \alpha}\left(-(t-s)^{\alpha} \lambda\right) f(s, y(s)) d s\right| \\
& \leq\left|\frac{1}{\Gamma(\alpha)} \int_{0}^{t}(t-s)^{\alpha-1} G d s\right| \leq \frac{t_{1}^{\alpha}}{\Gamma(\alpha+1)} \epsilon, \quad t \in\left[0, t_{1}\right] .
\end{aligned}
$$

\section{Existence and $\boldsymbol{\beta}$-Ulam-Hyers stability results}

We impose the following assumptions:

$\left(\mathrm{A}_{1}\right): f \in C(J \times \mathbb{R}, \mathbb{R})$.

$\left(\mathrm{A}_{2}\right)$ : There exists a positive constant $L_{f}$ such that

$$
\left|f\left(t, u_{1}\right)-f\left(t, u_{2}\right)\right| \leq L_{f}\left|u_{1}-u_{2}\right|, \quad \text { for each } t \in J \text { and all } u_{1}, u_{2} \in \mathbb{R} \text {. }
$$

$\left(\mathrm{A}_{3}\right): g_{i} \in C\left(\left[t_{i}, s_{i}\right] \times \mathbb{R}, \mathbb{R}\right)$ and there are positive constants $L_{g_{i}}, i=1,2, \ldots, m$ such that

$$
\left|g_{i}\left(t, u_{1}\right)-g_{i}\left(t, u_{2}\right)\right| \leq L_{g_{i}}\left|u_{1}-u_{2}\right|, \quad \text { for each } t \in\left[t_{i}, s_{i}\right] \text { and all } u_{1}, u_{2} \in \mathbb{R} \text {. }
$$

We begin by giving the existence and uniqueness result for the solutions to (1).

Theorem 4.1 Assume that $\left(\mathrm{A}_{1}\right),\left(\mathrm{A}_{2}\right),\left(\mathrm{A}_{3}\right)$ are satisfied. Then $(1)$ has a unique solution provided that

$$
\begin{aligned}
\varrho:= & \max \left\{\left(\frac{L_{f}\left(t_{i+1}-s_{i}\right)^{\alpha}}{\Gamma(\alpha+1)}\right)^{\beta}+\left(\frac{L_{g_{i}}\left(s_{i}-t_{i}\right)^{\gamma}}{\Gamma(\gamma+1)}\right)^{\beta}+\left(\frac{L_{f} s_{i}^{\alpha}}{\Gamma(\alpha+1)}\right)^{\beta},\right. \\
& \left.\left(\frac{L_{f} t_{1}^{\alpha}}{\Gamma(\alpha+1)}\right)^{\beta}: i=1,2, \ldots, m\right\}<1 .
\end{aligned}
$$

Proof Consider an operator $\Lambda: P C(J, \mathbb{R}) \rightarrow P C(J, \mathbb{R})$ defined by

$$
\begin{aligned}
(\Lambda x)(0)= & x_{0} \\
(\Lambda x)(t)= & q+I_{t_{i}, t}^{\gamma} g_{i}(t, x(t))-I_{0, s_{i}}^{\alpha} f\left(s_{i}, x\left(s_{i}\right)\right), \quad t \in\left(t_{i}, s_{i}\right], i=1,2, \ldots, m ; \\
(\Lambda x)(t)= & \mathbb{E}_{\alpha}\left(-t^{\alpha} \lambda\right) x_{0}+\int_{0}^{t}(t-s)^{\alpha-1} \mathbb{E}_{\alpha, \alpha}\left(-(t-s)^{\alpha} \lambda\right) f(s, x(s)) d s, \quad t \in\left[0, t_{1}\right] \\
(\Lambda x)(t)= & \int_{s_{i}}^{t}(t-s)^{\alpha-1} \mathbb{E}_{\alpha, \alpha}\left(-(t-s)^{\alpha} \lambda\right) f(s, x(s)) d s \\
& +\mathbb{E}_{\alpha}\left(-\left(t-s_{i}\right)^{\alpha} \lambda\right)\left(q+I_{t_{i}, s_{i}}^{\gamma} g_{i}\left(s_{i}, x\left(s_{i}\right)\right)-I_{0, s_{i}}^{\alpha} f\left(s_{i}, x\left(s_{i}\right)\right)\right) \\
& t \in\left(s_{i}, t_{i+1}\right], i=1,2, \ldots, m
\end{aligned}
$$

It is easy to see that $\Lambda$ is well defined. Next, we show that $\Lambda$ is a contraction mapping.

Case 1: For $u_{1}, u_{2} \in P C(J, \mathbb{R})$ and for each $t \in\left[0, t_{1}\right]$, we have

$$
\begin{aligned}
& \left|\left(\Lambda u_{1}\right)(t)-\left(\Lambda u_{2}\right)(t)\right| \\
& \quad=\mid \int_{0}^{t}(t-s)^{\alpha-1} \mathbb{E}_{\alpha, \alpha}\left(-(t-s)^{\alpha} \lambda\right) f\left(s, u_{1}(s)\right) d s
\end{aligned}
$$




$$
\begin{aligned}
& -\int_{0}^{t}(t-s)^{\alpha-1} \mathbb{E}_{\alpha, \alpha}\left(-(t-s)^{\alpha} \lambda\right) f\left(s, u_{2}(s)\right) d s \mid \\
\leq & \frac{L_{f}}{\Gamma(\alpha)} \int_{0}^{t}(t-s)^{\alpha-1}\left|u_{1}(s)-u_{2}(s)\right| d s \\
\leq & \frac{L_{f}}{\Gamma(\alpha)}\left\|u_{1}-u_{2}\right\|_{P C} \int_{0}^{t}(t-s)^{\alpha-1} d s \\
\leq & \frac{L_{f} t_{1}^{\alpha}}{\Gamma(\alpha+1)}\left\|u_{1}-u_{2}\right\|_{P C},
\end{aligned}
$$

which implies that

$$
\left|\left(\Lambda u_{1}\right)(t)-\left(\Lambda u_{2}\right)(t)\right|^{\beta} \leq\left(\frac{L_{f} t_{1}^{\alpha}}{\Gamma(\alpha+1)}\right)^{\beta}\left\|u_{1}-u_{2}\right\|_{P \beta} .
$$

This reduces to

$$
\left\|\Lambda u_{1}-\Lambda u_{2}\right\|_{P \beta} \leq\left(\frac{L_{f} t_{1}^{\alpha}}{\Gamma(\alpha+1)}\right)^{\beta}\left\|u_{1}-u_{2}\right\|_{P \beta} .
$$

Case 2: For $u_{1}, u_{2} \in P C(J, \mathbb{R})$ and for each $t \in\left(t_{i}, s_{i}\right], i=1,2, \ldots, m$, we have

$$
\begin{aligned}
\left|\left(\Lambda u_{1}\right)(t)-\left(\Lambda u_{2}\right)(t)\right| & \\
\leq & \frac{1}{\Gamma(\gamma)} \int_{t_{i}}^{t}(t-s)^{\gamma-1}\left|g_{i}\left(s, u_{1}(s)\right)-g_{i}\left(s, u_{2}(s)\right)\right| d s \\
& \quad+\frac{1}{\Gamma(\alpha)} \int_{0}^{s_{i}}\left(s_{i}-s\right)^{\alpha-1}\left|f\left(s, u_{1}(s)\right)-f\left(s, u_{2}(s)\right)\right| d s \\
\leq & \frac{L_{g_{i}}}{\Gamma(\gamma)} \int_{t_{i}}^{t}(t-s)^{\gamma-1}\left|u_{1}(s)-u_{2}(s)\right| d s+\frac{L_{f}}{\Gamma(\alpha)} \int_{0}^{s_{i}}\left(s_{i}-s\right)^{\alpha-1}\left|u_{1}(s)-u_{2}(s)\right| d s \\
\leq & \frac{L_{g_{i}}}{\Gamma(\gamma)}\left\|u_{1}-u_{2}\right\|_{P C} \int_{t_{i}}^{t}(t-s)^{\gamma-1} d s+\frac{L_{f}}{\Gamma(\alpha)}\left\|u_{1}-u_{2}\right\|_{P C} \int_{0}^{s_{i}}\left(s_{i}-s\right)^{\alpha-1} d s \\
\leq & \left(\frac{L_{g_{i}}\left(s_{i}-t_{i}\right)^{\gamma}}{\Gamma(\gamma+1)}+\frac{L_{f} s_{i}^{\alpha}}{\Gamma(\alpha+1)}\right)\left\|u_{1}-u_{2}\right\|_{P C},
\end{aligned}
$$

which implies that

$$
\left|\left(\Lambda u_{1}\right)(t)-\left(\Lambda u_{2}\right)(t)\right|^{\beta} \leq\left(\frac{L_{g_{i}}\left(s_{i}-t_{i}\right)^{\gamma}}{\Gamma(\gamma+1)}+\frac{L_{f} s_{i}^{\alpha}}{\Gamma(\alpha+1)}\right)^{\beta}\left\|u_{1}-u_{2}\right\|_{P \beta} .
$$

This reduces to

$$
\left\|\Lambda u_{1}-\Lambda u_{2}\right\|_{P \beta} \leq\left[\left(\frac{L_{g_{i}}\left(s_{i}-t_{i}\right)^{\gamma}}{\Gamma(\gamma+1)}\right)^{\beta}+\left(\frac{L_{f} s_{i}^{\alpha}}{\Gamma(\alpha+1)}\right)^{\beta}\right]\left\|u_{1}-u_{2}\right\|_{P \beta} .
$$

Case 3: For $u_{1}, u_{2} \in P C(J, \mathbb{R})$ and for each $t \in\left(s_{i}, t_{i+1}\right], i=1,2, \ldots, m$, we have

$$
\begin{aligned}
& \left|\left(\Lambda u_{1}\right)(t)-\left(\Lambda u_{2}\right)(t)\right| \\
& \quad \leq \frac{L_{f}}{\Gamma(\alpha)} \int_{s_{i}}^{t}(t-s)^{\alpha-1}\left|u_{1}(s)-u_{2}(s)\right| d s+\frac{L_{g_{i}}}{\Gamma(\gamma)} \int_{t_{i}}^{s_{i}}\left(s_{i}-s\right)^{\gamma-1}\left|u_{1}\left(s_{i}\right)-u_{2}\left(s_{i}\right)\right| d s
\end{aligned}
$$




$$
\begin{aligned}
& +\frac{L_{f}}{\Gamma(\alpha)} \int_{0}^{s_{i}}\left(s_{i}-s\right)^{\alpha-1}\left|u_{1}(s)-u_{2}(s)\right| d s \\
\leq & \frac{L_{f}\left(t_{i+1}-s_{i}\right)^{\alpha}}{\Gamma(\alpha+1)}\left\|u_{1}-u_{2}\right\|_{P C}+\frac{L_{g_{i}}\left(s_{i}-t_{i}\right)^{\gamma}}{\Gamma(\gamma+1)}\left\|u_{1}-u_{2}\right\|_{P C} \\
& +\frac{L_{f} s_{i}^{\alpha}}{\Gamma(\alpha+1)}\left\|u_{1}-u_{2}\right\|_{P C} \\
\leq & \left(\frac{L_{f}\left(t_{i+1}-s_{i}\right)^{\alpha}}{\Gamma(\alpha+1)}+\frac{L_{g_{i}}\left(s_{i}-t_{i}\right)^{\gamma}}{\Gamma(\gamma+1)}+\frac{L_{f} s_{i}^{\alpha}}{\Gamma(\alpha+1)}\right)\left\|u_{1}-u_{2}\right\|_{P C},
\end{aligned}
$$

which implies that

$$
\left|\left(\Lambda u_{1}\right)(t)-\left(\Lambda u_{2}\right)(t)\right|^{\beta} \leq\left(\frac{L_{f}\left(t_{i+1}-s_{i}\right)^{\alpha}}{\Gamma(\alpha+1)}+\frac{L_{g_{i}}\left(s_{i}-t_{i}\right)^{\gamma}}{\Gamma(\gamma+1)}+\frac{L_{f} s_{i}^{\alpha}}{\Gamma(\alpha+1)}\right)^{\beta}\left\|u_{1}-u_{2}\right\|_{P \beta} .
$$

This reduces to

$$
\begin{aligned}
& \left\|\Lambda u_{1}-\Lambda u_{2}\right\|_{P \beta} \\
& \quad \leq\left[\left(\frac{L_{f}\left(t_{i+1}-s_{i}\right)^{\alpha}}{\Gamma(\alpha+1)}\right)^{\beta}+\left(\frac{L_{g_{i}}\left(s_{i}-t_{i}\right)^{\gamma}}{\Gamma(\gamma+1)}\right)^{\beta}+\left(\frac{L_{f} s_{i}^{\alpha}}{\Gamma(\alpha+1)}\right)^{\beta}\right]\left\|u_{1}-u_{2}\right\|_{P \beta} .
\end{aligned}
$$

From the above cases, we obtain

$$
\left\|\Lambda u_{1}-\Lambda u_{2}\right\|_{P \beta} \leq \varrho\left\|u_{1}-u_{2}\right\|_{P \beta}
$$

where $\varrho$ is given in (6). Finally, we can deduce that $\Lambda$ is a contraction mapping. Then one can derive the result immediately.

In what follows, we discuss the stability of (1) by using the concept of $\beta$-Ulam-Hyers in the above section.

Theorem 4.2 With the same assumptions in Theorem 4.1. Then (1) is $\beta$-Ulam-Hyers stable with respect to $\epsilon$.

Proof Denote by $x$ the unique solution of

$$
\left\{\begin{array}{l}
{ }^{\mathrm{c}} D_{0, t}^{\alpha} x(t)=-\lambda x(t)+f(t, x(t)), \quad t \in\left(s_{i}, t_{i+1}\right], i=0,1,2, \ldots, m, \alpha \in(0,1), \\
x(t)=q+I_{t_{i}, t}^{\gamma} g_{i}(t, x(t))-I_{0, s_{i}}^{\alpha} f\left(s_{i}, x\left(s_{i}\right)\right), \quad t \in\left(t_{i}, s_{i}\right], i=1,2, \ldots, m, \\
x(0)=y(0) .
\end{array}\right.
$$

Then we get

$$
x(t)=\left\{\begin{array}{l}
q+I_{t_{i}, t}^{\gamma} g_{i}(t, x(t))-I_{0, s_{i}}^{\alpha} f\left(s_{i}, x\left(s_{i}\right)\right), \quad t \in\left(t_{i}, s_{i}\right], i=1,2, \ldots, m ; \\
\mathbb{E}_{\alpha}\left(-t^{\alpha} \lambda\right) y(0)+\int_{0}^{t}(t-s)^{\alpha-1} \mathbb{E}_{\alpha, \alpha}\left(-(t-s)^{\alpha} \lambda\right) f(s, x(s)) d s, \quad t \in\left(0, t_{1}\right] ; \\
\int_{s_{i}}^{t}(t-s)^{\alpha-1} \mathbb{E}_{\alpha, \alpha}\left(-(t-s)^{\alpha} \lambda\right) f(s, x(s)) d s \\
\quad+\mathbb{E}_{\alpha}\left(-\left(t-s_{i}\right)^{\alpha} \lambda\right)\left(q+I_{t_{i}, s_{i}}^{\gamma} g_{i}\left(s_{i}, x\left(s_{i}\right)\right)-I_{0, s_{i}}^{\alpha} f\left(s_{i}, x\left(s_{i}\right)\right)\right), \\
\quad t \in\left(s_{i}, t_{i+1}\right], i=1, \ldots, m .
\end{array}\right.
$$


Let $y \in P C^{1}(J, \mathbb{R})$ be a solution of the inequality (3). According to (4), for each $t \in\left(s_{i}, t_{i+1}\right]$, $i=1,2, \ldots, m$, we have

$$
\begin{aligned}
\mid y(t) & -\int_{0}^{t}(t-s)^{\alpha-1} \mathbb{E}_{\alpha, \alpha}\left(-(t-s)^{\alpha} \lambda\right) f(s, y(s)) d s \\
& -\mathbb{E}_{\alpha}\left(-\left(t-s_{i}\right)^{\alpha} \lambda\right)\left(q+I_{t_{i}, s_{i}}^{\gamma} g_{i}\left(s_{i}, y\left(s_{i}\right)\right)-I_{0, s_{i}}^{\alpha} f\left(s_{i}, y\left(s_{i}\right)\right)\right) \mid \\
\leq \epsilon & +\frac{\left(t_{i+1}-s_{i}\right)^{\alpha}}{\Gamma(\alpha+1)} \epsilon,
\end{aligned}
$$

and for $t \in\left(t_{i}, s_{i}\right], i=1,2, \ldots, m$, we have

$$
\left|y(t)-q-I_{t_{i}, t}^{\alpha} g_{i}(t, y(t))+I_{0, s_{i}}^{\alpha} f\left(s_{i}, y\left(s_{i}\right)\right)\right| \leq \epsilon,
$$

and for $t \in\left[0, t_{1}\right]$, we have

$$
\left|y(t)-\mathbb{E}_{\alpha}\left(-t^{\alpha} \lambda\right) y(0)-\int_{0}^{t}(t-s)^{\alpha-1} \mathbb{E}_{\alpha, \alpha}\left(-(t-s)^{\alpha} \lambda\right) f(s, y(s)) d s\right| \leq \frac{t_{1}^{\alpha}}{\Gamma(\alpha+1)} \epsilon .
$$

Case 1: For each $t \in\left[0, t_{1}\right]$, we get

$$
\begin{aligned}
|y(t)-x(t)| \leq & \left|y(t)-\mathbb{E}_{\alpha}\left(-t^{\alpha} \lambda\right) y(0)-\int_{0}^{t}(t-s)^{\alpha-1} \mathbb{E}_{\alpha, \alpha}\left(-(t-s)^{\alpha} \lambda\right) f(s, y(s)) d s\right| \\
& +\mid \int_{0}^{t}(t-s)^{\alpha-1} \mathbb{E}_{\alpha, \alpha}\left(-(t-s)^{\alpha} \lambda\right) f(s, y(s)) d s \\
& -\int_{0}^{t}(t-s)^{\alpha-1} \mathbb{E}_{\alpha, \alpha}\left(-(t-s)^{\alpha} \lambda\right) f(s, x(s)) d s \mid \\
\leq & \frac{t_{1}^{\alpha}}{\Gamma(\alpha+1)} \epsilon+\frac{L_{f}}{\Gamma(\alpha)} \int_{0}^{t}(t-s)^{\alpha-1}|y(s)-x(s)| d s \\
\leq & \frac{t_{1}^{\alpha}}{\Gamma(\alpha+1)} \epsilon+\frac{L_{f} t_{1}^{\alpha}}{\Gamma(\alpha+1)}\|y-x\|_{P C},
\end{aligned}
$$

where

$$
\begin{aligned}
|y(t)-x(t)|^{\beta} & \leq\left(\frac{t_{1}^{\alpha}}{\Gamma(\alpha+1)} \epsilon+\frac{L_{f} t_{1}^{\alpha}}{\Gamma(\alpha+1)}\|y-x\|_{P C}\right)^{\beta} \\
& \leq\left(\frac{t_{1}^{\alpha}}{\Gamma(\alpha+1)} \epsilon\right)^{\beta}+\left(\frac{L_{f} t_{1}^{\alpha}}{\Gamma(\alpha+1)}\right)^{\beta}\|y-x\|_{P \beta},
\end{aligned}
$$

which implies that

$$
\left[1-\left(\frac{L_{f} t_{1}^{\alpha}}{\Gamma(\alpha+1)}\right)^{\beta}\right]\|y-x\|_{P \beta} \leq\left(\frac{t_{1}^{\alpha}}{\Gamma(\alpha+1)} \epsilon\right)^{\beta} .
$$

Thus,

$$
|y(t)-x(t)|^{\beta} \leq c_{f, \alpha, \gamma, \beta, g_{i}} \epsilon^{\beta}, \quad t \in\left[0, t_{1}\right],
$$


where

$$
c_{f, \alpha, \gamma, \beta, g_{i}}:=\frac{\left(\frac{t_{1}^{\alpha}}{\Gamma(\alpha+1)}\right)^{\beta}}{1-\left(\frac{L_{f} t_{1}^{\alpha}}{\Gamma(\alpha+1)}\right)^{\beta}} .
$$

Case 2: For $t \in\left(t_{i}, s_{i}\right], i=1,2, \ldots, m$, we have

$$
\begin{aligned}
|y(t)-x(t)|^{\beta} \leq & \left|y(t)-q-I_{t_{i},}^{\gamma} g_{i}(t, y(t))-I_{0, s_{i}}^{\alpha} f\left(s_{i}, y\left(s_{i}\right)\right)\right|^{\beta} \\
& +\left|I_{0, s_{i}}^{\alpha} f\left(s_{i}, y\left(s_{i}\right)\right)-I_{0, s_{i}}^{\alpha} f\left(s_{i}, x\left(s_{i}\right)\right)\right|^{\beta} \\
& +\left|I_{t_{i}}^{\gamma}, t_{i}(t, y(t))-I_{t_{i}, t}^{\gamma} g_{i}(t, x(t))\right|^{\beta} \\
\leq & \epsilon^{\beta}+\left[\left(\frac{L_{f} s_{i}^{\alpha}}{\Gamma(\alpha+1)}\right)^{\beta}+\left(\frac{L_{g_{i}}\left(s_{i}-t_{i}\right)^{\gamma}}{\Gamma(\gamma+1)}\right)^{\beta}\right]\|y-x\|_{P \beta},
\end{aligned}
$$

which implies that

$$
\left[1-\left(\frac{L_{f} s_{i}^{\alpha}}{\Gamma(\alpha+1)}\right)^{\beta}-\left(\frac{L_{g_{i}}\left(s_{i}-t_{i}\right)^{\gamma}}{\Gamma(\gamma+1)}\right)^{\beta}\right]\|y-x\|_{P \beta} \leq \epsilon^{\beta} .
$$

Thus,

$$
|y(t)-x(t)|^{\beta} \leq c_{f, \alpha, \gamma, \beta, g_{i}} \epsilon^{\beta}, \quad t \in\left(t_{i}, s_{i}\right], i=1,2, \ldots, m,
$$

where

$$
c_{f, \alpha, \gamma, \beta, g_{i}}:=\frac{1}{1-\left(\frac{L_{f} s_{i}^{\alpha}}{\Gamma(\alpha+1)}\right)^{\beta}-\left(\frac{L_{g_{i}}\left(s_{i}-t_{i}\right)^{\gamma}}{\Gamma(\gamma+1)}\right)^{\beta}} .
$$

Case 3: For $t \in\left(s_{i}, t_{i+1}\right], i=1,2, \ldots, m$, we have

$$
\begin{aligned}
|y(t)-x(t)|^{\beta} \leq & \mid y(t)-\int_{s_{i}}^{t}(t-s)^{\alpha-1} \mathbb{E}_{\alpha, \alpha}\left(-(t-s)^{\alpha} \lambda\right) f(s, y(s)) d s \\
& -\left.\mathbb{E}_{\alpha}\left(-\left(t-s_{i}\right)^{\alpha} \lambda\right)\left(q+I_{t_{i}, s_{i}}^{\gamma} g_{i}\left(s_{i}, y\left(s_{i}\right)\right)-I_{0, s_{i}}^{\alpha} f\left(s_{i}, y\left(s_{i}\right)\right)\right)\right|^{\beta} \\
& +\left[\frac{1}{\Gamma(\alpha)} \int_{0}^{t}(t-s)^{\alpha-1}|f(s, y(s))-f(s, x(s))| d s\right]^{\beta} \\
& +\left|I_{t_{i}, s_{i}}^{\gamma} g_{i}\left(s_{i}, y\left(s_{i}\right)\right)-I_{t_{i}, s_{i}}^{\gamma} g_{i}\left(s_{i}, x\left(s_{i}\right)\right)\right|^{\beta}+\left|I_{0, s_{i}}^{\alpha} f\left(s_{i}, y\left(s_{i}\right)\right)-I_{0, s_{i}}^{\alpha} f\left(s_{i}, x\left(s_{i}\right)\right)\right|^{\beta} \\
\leq & \left(\epsilon+\frac{\left(t_{i+1}-s_{i}\right)^{\alpha}}{\Gamma(\alpha+1)} \epsilon\right)^{\beta} \\
& +\left[\left(\frac{L_{f}\left(t_{i+1}-s_{i}\right)^{\alpha}}{\Gamma(\alpha+1)}\right)^{\beta}+\left(\frac{L_{g_{i}}\left(s_{i}-t_{i}\right)^{\gamma}}{\Gamma(\gamma+1)}\right)^{\beta}+\left(\frac{L_{f} s_{i}^{\alpha}}{\Gamma(\alpha+1)}\right)^{\beta}\right]\|y-x\|_{P \beta},
\end{aligned}
$$

which yields

$$
\begin{aligned}
& \left\{1-\left[\left(\frac{L_{f}\left(t_{i+1}-s_{i}\right)^{\alpha}}{\Gamma(\alpha+1)}\right)^{\beta}+\left(\frac{L_{g_{i}}\left(s_{i}-t_{i}\right)^{\gamma}}{\Gamma(\gamma+1)}\right)^{\beta}+\left(\frac{L_{f} s_{i}^{\alpha}}{\Gamma(\alpha+1)}\right)^{\beta}\right]\right\}\|y-x\|_{P \beta} \\
& \leq\left(\epsilon+\frac{\left(t_{i+1}-s_{i}\right)^{\alpha}}{\Gamma(\alpha+1)} \epsilon\right)^{\beta} \leq\left[1+\left(\frac{\left(t_{i+1}-s_{i}\right)^{\alpha}}{\Gamma(\alpha+1)}\right)^{\beta}\right] \epsilon^{\beta} .
\end{aligned}
$$


Thus,

$$
|y(t)-x(t)|^{\beta} \leq c_{f, \alpha, \gamma, \beta, g_{i}} \epsilon^{\beta}, \quad t \in\left(s_{i}, t_{i+1}\right], i=1,2, \ldots, m,
$$

where

$$
c_{f, \alpha, \gamma, \beta, g_{i}}:=\frac{1+\left(\frac{\left(t_{i+1}-s_{i}\right)^{\alpha}}{\Gamma(\alpha+1)}\right)^{\beta}}{1-\left[\left(\frac{L_{f}\left(t_{i+1}-s_{i}\right)^{\alpha}}{\Gamma(\alpha+1)}\right)^{\beta}+\left(\frac{\left.L_{g_{i}} s_{i}-t_{i}\right)^{\gamma}}{\Gamma(\gamma+1)}\right)^{\beta}+\left(\frac{L_{f} s_{i}^{\alpha}}{\Gamma(\alpha+1)}\right)^{\beta}\right]} .
$$

Summarizing, (8), (9), and (10) imply that (1) is $\beta$-Ulam-Hyers stable with respect to $\epsilon$. The proof is completed.

\section{Example}

Let us consider

$$
\left\{\begin{array}{l}
{ }^{\mathrm{c}} D_{0, t}^{\frac{3}{5}} x(t)=-x(t)+\frac{1}{8+e^{t}+t^{2}} \arctan \left(t^{2}+x(t)\right), \quad t \in(0,1] \\
x(t)=q+\frac{1}{\Gamma\left(\frac{2}{3}\right)} \int_{1}^{t}(t-s)^{-\frac{1}{3}} \frac{|x(s)|}{16(1+|x(s)|)} d s \\
\quad-\frac{1}{\Gamma\left(\frac{3}{5}\right)} \int_{0}^{2}(2-s)^{-\frac{2}{5}} \frac{1}{8+e^{s}+s^{2}} \arctan \left(s^{2}+x(s)\right) d s, \quad t \in(1,2]
\end{array}\right.
$$

and

$$
\left\{\begin{array}{l}
\left|{ }^{\mathrm{c}} D_{0, t}^{\frac{3}{5}} y(t)+y(t)-\frac{1}{8+e^{t}+t^{2}} \arctan \left(t^{2}+y(t)\right)\right| \leq 1, \quad t \in(0,1] \\
\mid y(t)-q-\frac{1}{\Gamma\left(\frac{2}{3}\right)} \int_{1}^{t}(t-s)^{-\frac{1}{3}} \frac{|y(s)|}{16(1+|y(s)|)} d s \\
\quad+\frac{1}{\Gamma\left(\frac{3}{5}\right)} \int_{0}^{2}(2-s)^{-\frac{2}{5}} \frac{1}{8+e^{s}+s^{2}} \arctan \left(s^{2}+y(s)\right) d s \mid \leq 1, \quad t \in(1,2] .
\end{array}\right.
$$

Set $\lambda=1, \alpha=\frac{3}{5}, \gamma=\frac{2}{3}, J=[0,2], 0=t_{0}=s_{0}<t_{1}=1<s_{1}=2$, and $\beta=\frac{1}{2}$. Denote $f(t, x(t))=$ $\frac{1}{8+e^{t}+t^{2}} \arctan \left(t^{2}+x(t)\right)$ with $L_{f}=\frac{1}{9}$ for $t \in(0,1]$ and $I_{1, t}^{\frac{2}{3}} g_{1}(t, x(t))=\frac{1}{\Gamma\left(\frac{2}{3}\right)} \int_{1}^{t}(t-s)^{-\frac{1}{3}} \frac{|x(s)|}{16(1+|x(s)|)} d s$ with $L_{g_{1}}=\frac{1}{16}$ for $t \in(1,2]$. Moreover, we put $\epsilon=1$.

Let $y \in P C^{1}([0,2], \mathbb{R})$ be a solution of the inequality (12). Then there exists $G \in \mathbb{R}$ such that $|G| \leq 1$ and

$$
\begin{aligned}
{ }^{\mathrm{c}} D_{0, t}^{\frac{3}{5}} y(t) & =-y(t)+\frac{1}{8+e^{t}+t^{2}} \arctan \left(t^{2}+y(t)\right)+G, \quad t \in(0,1], \\
y(t)= & q+\frac{1}{\Gamma\left(\frac{2}{3}\right)} \int_{1}^{t}(t-s)^{-\frac{1}{3}} \frac{|y(s)|}{16(1+|y(s)|)} d s \\
& -\frac{1}{\Gamma\left(\frac{3}{5}\right)} \int_{0}^{2}(2-s)^{-\frac{2}{5}} \frac{1}{8+e^{s}+s^{2}} \arctan \left(s^{2}+y(s)\right) d s+G, \quad t \in(1,2] .
\end{aligned}
$$

For $t \in(0,1]$, integrating (13) from 0 to $t$, we have

$$
\begin{aligned}
y(t)= & \mathbb{E}_{\frac{3}{5}}\left(-t^{\frac{3}{5}}\right) y(0) \\
& +\int_{0}^{t}(t-s)^{\frac{3}{5}-1} \mathbb{E}_{\frac{3}{5}, \frac{3}{5}}\left(-(t-s)^{\frac{3}{5}}\right)\left(\frac{1}{8+e^{s}+s^{2}} \arctan \left(s^{2}+y(s)\right)+G\right) d s .
\end{aligned}
$$


For $t \in(1,2]$, we have

$$
\begin{aligned}
y(t)= & q+\frac{1}{\Gamma\left(\frac{2}{3}\right)} \int_{1}^{t}(t-s)^{-\frac{1}{3}} \frac{|y(s)|}{16(1+|y(s)|)} d s \\
& -\frac{1}{\Gamma\left(\frac{3}{5}\right)} \int_{0}^{2}(2-s)^{-\frac{2}{5}} \frac{1}{8+e^{s}+s^{2}} \arctan \left(s^{2}+y(s)\right) d s+G .
\end{aligned}
$$

After checking the conditions in Theorem 4.1, we find that

$$
\left\{\begin{array}{l}
{ }^{\mathrm{c}} D_{0, t}^{\frac{3}{5}} x(t)=-x(t)+\frac{1}{8+e^{t}+t^{2}} \arctan \left(t^{2}+x(t)\right), \quad t \in(0,1], \\
x(t)=q_{1}+\frac{1}{\Gamma\left(\frac{2}{3}\right)} \int_{1}^{t}(t-s)^{-\frac{1}{3}} \frac{|x(s)|}{16(1+|x(s)|)} d s \\
\quad-\frac{1}{\Gamma\left(\frac{3}{5}\right)} \int_{0}^{2}(2-s)^{-\frac{2}{5}} \frac{1}{8+e^{s}+s^{2}} \arctan \left(s^{2}+x(s)\right) d s, \quad t \in(1,2], \\
x(0)=y(0),
\end{array}\right.
$$

has a unique solution, where

$$
\varrho:=\max \left\{\frac{1}{3 \sqrt{\Gamma\left(\frac{8}{5}\right)}}, \frac{1}{4 \sqrt{\Gamma\left(\frac{5}{3}\right)}}+\frac{2^{\frac{3}{10}}}{3 \sqrt{\Gamma\left(\frac{8}{5}\right)}}\right\} \approx \max \{0.3526,0.6972\}<1 .
$$

Next, let us take the solution $x$ of the problem (14) given by

$$
\begin{aligned}
x(t)= & \mathbb{E}_{\frac{3}{5}}\left(-t^{\frac{3}{5}}\right) y(0) \\
& +\int_{0}^{t}(t-s)^{-\frac{2}{5}} \mathbb{E}_{\frac{3}{5}, \frac{3}{5}}\left(-(t-s)^{\frac{3}{5}}\right)\left(\frac{1}{8+e^{s}+s^{2}} \arctan \left(s^{2}+x(s)\right)\right) d s, \quad t \in(0,1], \\
x(t)= & q+\frac{1}{\Gamma\left(\frac{2}{3}\right)} \int_{1}^{t}(t-s)^{-\frac{1}{3}} \frac{|x(s)|}{16(1+|x(s)|)} d s \\
& -\frac{1}{\Gamma\left(\frac{3}{5}\right)} \int_{0}^{2}(2-s)^{-\frac{2}{5}} \frac{1}{8+e^{s}+s^{2}} \arctan \left(s^{2}+x(s)\right) d s, \quad t \in(1,2] .
\end{aligned}
$$

For $t \in(0,1]$, we have

$$
|y(t)-x(t)|^{\frac{1}{2}} \leq \frac{\frac{1}{\sqrt{\Gamma\left(\frac{8}{5}\right)}}}{1-\frac{1}{3 \sqrt{\Gamma\left(\frac{8}{5}\right)}}} \epsilon^{\frac{1}{2}} \leq 1.64
$$

For $t \in(1,2]$, we have

$$
|y(t)-x(t)|^{\frac{1}{2}} \leq \frac{1}{1-\frac{2 \frac{3}{10}}{3 \sqrt{\Gamma\left(\frac{8}{5}\right)}}-\frac{1}{4 \sqrt{\Gamma\left(\frac{5}{3}\right)}}} \leq 3.3 .
$$

Summarizing, we have

$$
|y(t)-x(t)|^{\frac{1}{2}} \leq 3.3=3.3 \cdot 1^{\frac{1}{2}}, \quad t \in J,
$$

which shows that (11) is $\frac{1}{2}$-Ulam-Hyers stable with respect to $\epsilon=1$. 


\section{Conclusions}

This paper has investigated a new class of fractional differential equations with instantaneous impulses. In particular, the existence and $\beta$-Ulam-Hyers stability for such a new class of impulsive equations on a compact interval are obtained.

Competing interests

The author declares to have no competing interests.

\section{Author's contributions}

YXL proved the theorems, interpreted the results, wrote the article, and defined the research theme, and read and approved the manuscript.

\section{Acknowledgements}

The author thanks the referees for their careful reading of the manuscript and insightful comments, which helped to improve the quality of the paper. The author would also like to acknowledge the valuable comments and suggestions from the editors, which vastly contributed to improvement of the presentation of the paper. This work is partially supported by Youth Science Foundation of Shanxi University of Finance and Economics $(2014026,2014003)$.

Received: 6 September 2014 Accepted: 12 February 2015 Published online: 31 March 2015

\section{References}

1. Agarwal, RP, Benchohra, M, Hamani, S: A survey on existence results for boundary value problems of nonlinear fractional differential equations and inclusions. Acta Appl. Math. 109, 973-1033 (2010)

2. Benchohra, M, Seba, D: Impulsive fractional differential equations in Banach spaces. Electron. J. Qual. Theory Differ. Equ. Spec. Ed. I 2009, 8 (2009)

3. Fečkan, M, Zhou, Y, Wang, J: On the concept and existence of solution for impulsive fractional differential equations. Commun. Nonlinear Sci. Numer. Simul. 17, 3050-3060 (2012)

4. Rehman, M, Eloe, PW: Existence and uniqueness of solutions for impulsive fractional differential equations. Appl. Math. Comput. 224, 422-431 (2013)

5. Hernández, E, O’Regan, D: On a new class of abstract impulsive differential equations. Proc. Am. Math. Soc. 141, 1641-1649 (2013)

6. Pierri, $M, O^{\prime}$ Regan, D, Rolnik, V: Existence of solutions for semi-linear abstract differential equations with not instantaneous impulses. Appl. Math. Comput. 219, 6743-6749 (2013)

7. Tarasov, VE: Fractional Dynamics: Application of Fractional Calculus to Dynamics of Particles, Fields and Media. Springer, Berlin (2010)

8. Baleanu, D, Diethelm, K, Scalas, E, Trujillo, JJ: Fractional Calculus Models and Numerical Methods. Series on Complexity, Nonlinearity and Chaos. World Scientific, Singapore (2012)

9. Ulam, SM: A Collection of Mathematical Problems. Interscience, New York (1968)

10. Hyers, DH, Isac, G, Rassias, TM: Stability of Functional Equations in Several Variables. Birkhäuser, Basel (1998)

11. Rassias, TM: On the stability of linear mappings in Banach spaces. Proc. Am. Math. Soc. 72, 297-300 (1978)

12. Jung, S-M: Hyers-Ulam-Rassias Stability of Functional Equations in Nonlinear Analysis. Springer, New York (2011)

13. Cădariu, L: Stabilitatea Ulam-Hyers-Bourgin pentru ecuatii functionale. Ed. Univ. Vest Timişoara, Timişoara (2007)

14. Jung, S-M, Kim, TS, Lee, KS: A fixed point approach to the stability of quadratic functional equation. Bull. Korean Math. Soc. 43, 531-541 (2006)

15. András, S, Mészáros, AR: Ulam-Hyers stability of dynamic equations on time scales via Picard operators. Appl. Math. Comput. 219, 4853-4864 (2013)

16. Burger, M, Ozawa, N, Thom, A: On Ulam stability. Isr. J. Math. 193, 109-129 (2013)

17. Lungu, N, Popa, D: Hyers-Ulam stability of a first order partial differential equation. J. Math. Anal. Appl. 385, 86-91 (2012)

18. Popa, D, Raşa, I: On the Hyers-Ulam stability of the linear differential equation. J. Math. Anal. Appl. 381, $530-537$ (2011)

19. Rus, IA: Ulam stabilities of ordinary differential equations in a Banach space. Carpath. J. Math. 26, 103-107 (2010)

20. Wang, J, Fečkan, M, Zhou, Y: Ulam's type stability of impulsive ordinary differential equations. J. Math. Anal. Appl. 395, 258-264 (2012)

21. Xu, TZ: On the stability of multi-Jensen mappings in $\beta$-normed spaces. Appl. Math. Lett. 25, 1866-1870 (2012)

22. Ciepliński, K: Stability of multi-additive mappings in $\beta$-Banach spaces. Nonlinear Anal. TMA 75, 4205-4212 (2012)

23. Kilbas, AA, Srivastava, HM, Trujillo, JJ: Theory and Applications of Fractional Differential Equations. Elsevier, Amsterdam (2006)

24. Wang, J, Fečkan, M, Zhou, Y: Presentation of solutions of impulsive fractional Langevin equations and existence results. Eur. Phys. J. Spec. Top. 222, 1855-1872 (2013) 